\title{
A Novel Paper Currency Recognition using Fourier Mellin Transform, Hidden Markov Model and Support Vector Machine
}

\author{
Abbas Yaseri \\ Hadaf Institute of Higher Education
}

\author{
Seyed Mahmoud Anisheh \\ Hadaf Institute of Higher Education
}

\begin{abstract}
A paper currency recognition system has a wide range of applications such as self receiver machines for automated teller machines and automatic good-selling machines. In this paper a new paper currency recognition system based on Fourier-Mellin transform, Markovian characteristics and Support Vector Machine (SVM) is presented. In the first, a pre-processing algorithm by Fourier-Mellin transform is performed. The key feature of Fourier-Mellin transform is that it is invariant in rotation, translation and scale of the input image. Then, obtained image is segmented and markovian characteristics of each segment have been utilized to construct a feature vectors. These vectors are then fed into SVM classifier for paper currency recognition. In order to evaluate the effectiveness of the system several experiments are carried out. Experimental result indicates that the proposed method achieved high accuracy rate in paper currency recognition.
\end{abstract}

Keywords Paper currency recognition, Fourier-Mellin Transform, Markovian characteristics, Support Vector Machine.

\section{Introduction}

By development of modern banking services, applications of automatic paper currency recognition methods can be found in different fields such as automated teller machines and automatic goods-seller machines. The needs for an automatic banknote recognition system encouraged many researchers to develop fast, accurate, reliable and robust technique [1]. In addition, the proposed system should also be able to adapt to high noise, which is a critical factor. Hence, the task at hand to be solved can be categorized into two components [2]. The first step includes extracting features of the paper currency images that vary from each denomination. The second step requires using these features in an intelligent system for recognition.

Paper currency recognition systems should be able to recognize banknotes from each side and each direction. Since banknotes may be defected during circulation, the designed system should have a high accuracy in detecting torn or worn banknotes.

Automatic currency note recognition highly depends on the currency note characteristics of a particular country and the extraction of features directly affects the recognition ability. Therefore, these methods can be used only for recognition of a few banknote denominations, for example, the banknotes of one country.

In this paper a new paper currency recognition is presented. The proposed technique is independent to the number of paper currency classes. In this paper, texture characteristics of banknotes are used for paper currency recognition. The Hidden Markov Model (HMM) is utilized to model texture of the banknotes as a random process. The features employed in this paper are independent to the way that a paper currency is placed in front of the sensor.

This paper is organized as follows: the existing paper currency recognition methods are described in Section 2. Section 3 presents the background knowledge for the proposed method. The proposed paper currency recognition method is explained in Sections 4. The performance evaluation of the proposed method and comparison results are provided in Section 5. Section 6 summarizes our conclusions.

\section{Existing paper currency recognition Methods}

There are a number of paper currency recognition methods in the literature. In [3, 4], symmetrical masks has been used for recognizing paper currency in any direction. In this method, the summation of non-masked pixel values in each banknote is computed and fed to a neural network for recognizing paper currency. In this method, two sensors are used for recognition of the front and back of the paper currency, but the image of the front is the only criterion for decision. In another research [5], the edges of patterns on a paper currency are initially detected. In the next step, paper currency is divided into $N$ equal parts along vertical vector. Then, for each edge of the obtained parts the number of pixels are counted and fed to a three-layer, back propagation neural network. In this method, to overcome the problem of recognizing dirty worn banknotes, the following linear function is used as a pre-processing step:

$f(x)=F_{a} x+F_{b}$

where $x$ is the input image in gray scale, $f(x)$ is the output image; and $F_{a}, F_{b}$ and $\mathrm{N}$ are selected $3,-128$ and 50, respectively.

In [2] the size and the color of paper currency are considered as the first step in paper currency recognition. Then, HMM has been utilized as a robust technique for texture feature extraction and recognizing all of paper currency in the world. It is clear that texture recognition algorithm must have a lowexecution time. Therefore, to reduce the computational load, reducing the gray scale levels of the banknote images has been proposed.

In [6] the authors developed a paper currency recognition method proposed in [2]. However, the mentioned method is relatively sensitive to translation, rotation and scale of the original image. In order to overcome of this problem we proposed a new method based on Fourier-Mellin transform. In addition, our experimental results indicate that we can achieve 
a higher paper currency recognition rate using SVM classifier compared to method proposed in [6].

\section{Background knowledge for the proposed method}

Since this research is based on wiener filter, Fourier-Mellin transfotm, HMM and SVM, they are briefly reviewed here.

\subsection{Wiener Filter}

To improve the accuracy of the proposed method in recognizing dirty banknotes, it is necessary to pass the paper currency through a filter in order to reduce the effect of dirt through improving the lightness of the image. In fact, a dirty paper currency is the one that the darkness of its image was increased. The important point is that the severity of the increased darkness is almost the same on the whole surface of the paper currency. In order to reduce the effect of dirt on the image using a wiener filter could be very helpful. Wiener filter estimates the local mean and variance around each pixel as described below [7, 8]:

$\mu=\frac{1}{M N} \sum_{n 1, n 2 \in(N \times M)} \alpha\left(n_{1}, n_{2}\right)$

$\sigma^{2}=\frac{1}{M N} \sum_{n 1, n 2 \in(N \times M)} \alpha^{2}\left(n_{1}, n_{2}\right)-\mu^{2}$

where $\alpha$ is the N-by-M local neighborhood of each pixel in the image [7,8] Then, a pixel-wise Wiener filter using the above estimation has been created:

$b\left(n_{1}, n_{2}\right)=\mu+\frac{\sigma^{2}-v^{2}}{\sigma^{2}}\left[\alpha\left(n_{1}, n_{2}\right)-\mu\right]$

where $v^{2}$ is the noise variance. If the noise variance is not given, Wiener uses the average of all the local estimated variances. This approach often provides a better result, preserving edges and other high-frequency parts of an image, compared to linear filtering [7]. Figure 1. shows the results of applying the Wiener filter on10 euro clean and worn banknote. As the figure shows, applying the Wiener filter reduces the dirt from the worn banknote.

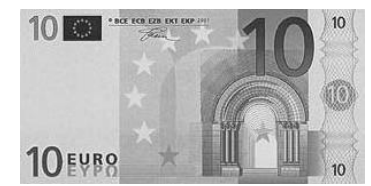

(a)

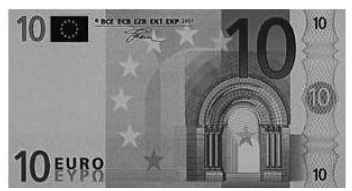

(c)

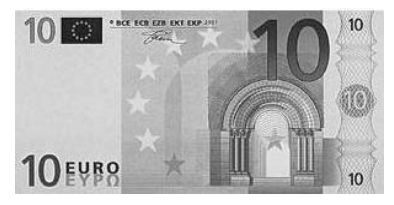

(b)

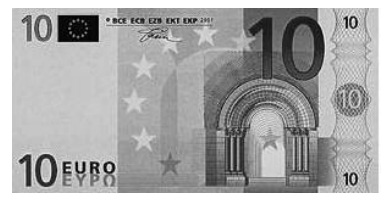

(d)
Fig 1:Using the Wiener filter to reduce the dirt from the worn banknote.

a)Original image before filtering, b)Obtained image after filtering.

c)Worn image before filtering

d)Worn image after filtering.

\subsection{Fourier-Mellin Transform}

The Fourier-Mellin transform is a powerful tool for image recognition because its resulting spectrum is invariant in rotation, translation and scale [9]. The Fourier Transform (FT) itself is translation invariant and its conversion to log-polar coordinates converts the scale and rotation differences to vertical and horizontal offsets that can be measured. A second FFT, called the Mellin transform gives a transform-space image that is invariant to translation, rotation and scale [10]. For an input image, $\mathrm{I}[\mathrm{m}, \mathrm{n}]$, the Fourier-Mellin transform is defined as below:

$|F\{I[m, n]\}|=|F[k, l]|=$

$\left|\sum_{m=0}^{M-1} \sum_{n=0}^{N-1} I[m, n] e^{-j\left(\frac{2 \pi}{M}\right)(k m)} e^{-j\left(\frac{2 \pi}{N}\right)(\mathrm{ln})}\right|$

$m_{0}=\frac{N}{2}, \quad n_{0}=\frac{N}{2} \quad$ for $\quad N \quad$ odd

$m_{0}=\frac{(N-1)}{2}, \quad n_{0}=\frac{(N-1)}{2}$ for $N$

Figure 2 shows the results of applying the Fourier-Mellin Transform on rotated banknote. As the figure shows, applying the Fourier-Mellin Transform reduces the effect of the rotation of the banknote.

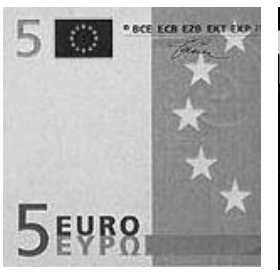

(a)

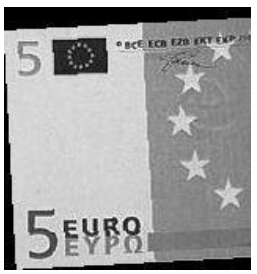

(b)

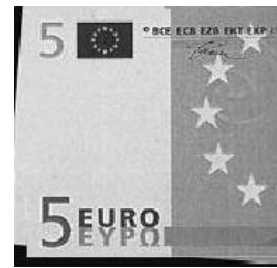

(c)
Fig 2:Using the Fourier-Mellin transform to reduce the effect of rotation of the banknote:

a) Original image, b) Rotated image

c) Output image after applying Fourier-Mellin transform

\subsection{Hidden Markov Model (HMM)}

By using the Hidden Markov Model (HMM) in this approach, the texture of a banknote is modeled as a random process. A random process $\left\{x_{n}, n=0,1,2, \ldots\right\}$ is called a Markov chain if the possibility value in state $x_{n+1}$ depends on just the possible value in state $x_{n}$ [11] that is:

$$
P=\left[\begin{array}{cccc}
p_{11} & p_{12} & \ldots & p_{1 n} \\
p_{21} & p_{22} & \ldots & p_{2 n} \\
\cdot & \cdot & & \cdot \\
\cdot & \cdot & & \cdot \\
\cdot & \cdot & & \cdot \\
p_{n 1} & p_{n 2} & \ldots & p_{n n}
\end{array}\right]
$$


where $\mathrm{n}$ is the number of states in a chain. In a discrete time Markov chain, the possibility value of different states in the matrix is computed as follow:

$$
p_{i j}=\frac{n_{i j}}{\sum_{k=1}^{n} n_{i k}}
$$

Where $n_{i j}$ is the number of transitions from state $i$ to state $j$. Considering equation (6), matrix $P$ can be multiplied by the factor $\sum_{k=1}^{n} n_{i k}$

In order to obtain equation (7), this matrix is used to differentiate between textures in different denominations. We can scan the banknotes from top to bottom and from left to right to obtain the transition matrix across the row $\left(N_{x}\right)$ and across the column $\left(N_{y}\right)$.

$$
N=p_{i j} \sum_{k=1}^{n} n_{i k}=\left[\begin{array}{cccc}
N_{11} & N_{12} & \ldots & N_{1 n} \\
N_{21} & N_{22} & \ldots & N_{2 n} \\
\cdot & \cdot & & \cdot \\
\cdot & \cdot & & \cdot \\
\cdot & \cdot & & \cdot \\
N_{n 1} & N_{n 2} & \ldots & N_{n n}
\end{array}\right]
$$

An image is recognized by the value of its pixels at different places, the way that adjacent pixels vary can also be used to distinguish different images [12]. Considering a paper currency image as an $l_{m} \times n$ matrix that is shown in table1, the value of each pixel like ij can be considered as one state.

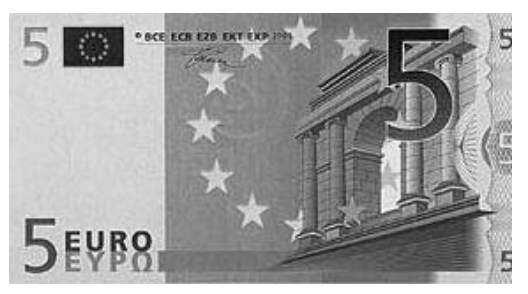

Fig 3: Image of 5 Euro banknote

It is clear that using the lower numbers of gray scales leads to a lower computational load. Therefore, in this paper the main diagonal values of the matrix $\mathrm{N}$ have been used. Each element of the main diagonal value represents the number of times that the corresponding value repeated in adjacent pixels. The transition matrix $(\mathrm{N})$ for a 5 euro banknote shown in Figure 3 that has been quantized in 11 level gray scales is shown in Table 1. In this table, for example, the 39 represents the number of times the values of adjacent pixels are between 0 and 22. Our investigations indicate that the main diagonal values are sufficient to distinguish different denominations. The main diagonal values of seven different euro banknotes are illustrated in Table 2. As it shows, all of the banknotes are distinguishable using the main diagonal value. It should be noted that the dominant colors of the banknote is recognizable using the main diagonal values. Our investigations indicate that using the main diagonal values is robust against worn banknotes. To show the robustness of this feature, the main diagonal values of clean and dirty 5 euro banknote are shown in table3. Because of the monotonic impurity in dirty banknotes, the effect of the impurity on the main diagonal values is negligible.

Table 1.

Transition matrix for a 5 Euro banknote

\begin{tabular}{|l|l|l|l|l|l|l|l|l|l|l|}
\hline 45 & 19 & 16 & 8 & 7 & 5 & 5 & 4 & 4 & 0 & 0 \\
\hline 35 & 402 & 238 & 50 & 38 & 25 & 9 & 16 & 21 & 4 & 0 \\
\hline 9 & 235 & 871 & 356 & 114 & 90 & 48 & 25 & 15 & 9 & 5 \\
\hline 7 & 50 & 337 & 1553 & 459 & 183 & 116 & 39 & 33 & 17 & 5 \\
\hline 3 & 29 & 150 & 556 & 1938 & 700 & 231 & 100 & 74 & 35 & 12 \\
\hline 2 & 26 & 59 & 157 & 876 & 7077 & 1726 & 240 & 125 & 70 & 28 \\
\hline 5 & 19 & 49 & 51 & 222 & 1860 & 3406 & 467 & 213 & 126 & 26 \\
\hline 6 & 52 & 26 & 26 & 75 & 256 & 486 & 793 & 675 & 331 & 39 \\
\hline 1 & 5 & 13 & 23 & 46 & 107 & 198 & 634 & 6726 & 2098 & 60 \\
\hline 0 & 1 & 15 & 17 & 48 & 67 & 198 & 409 & 1978 & 3769 & 80 \\
\hline 0 & 0 & 3 & 2 & 5 & 16 & 21 & 38 & 67 & 103 & 49 \\
\hline
\end{tabular}


Table 2.

Transition matrix for 7 types Euro banknotes

\begin{tabular}{|l|l|l|l|l|l|l|l|l|l|l|l|}
\hline 5euro & 45 & 402 & 871 & 1553 & 1938 & 7077 & 3406 & 793 & 6726 & 3769 & 49 \\
\hline 10euro & 0 & 35 & 609 & 585 & 512 & 2284 & 2646 & 6804 & 12852 & 2760 & 5 \\
\hline 20euro & 9 & 451 & 1359 & 461 & 1007 & 4821 & 2025 & 5372 & 11825 & 245 & 8 \\
\hline 50euro & 0 & 261 & 985 & 777 & 2122 & 4753 & 6051 & 6806 & 19935 & 16754 & 19969 \\
\hline 100euro & 0 & 119 & 1405 & 910 & 1430 & 2503 & 5725 & 5648 & 8204 & 1427 & 34 \\
\hline 200euro & 0 & 83 & 811 & 768 & 678 & 913 & 1605 & 3287 & 5160 & 15485 & 90 \\
\hline 500euro & 0 & 98 & 1255 & 876 & 1429 & 2615 & 8082 & 2457 & 2412 & 8895 & 63 \\
\hline
\end{tabular}

Table 3.

Transition matrix for original 5 Euro and dirty 5 euro

\begin{tabular}{|l|l|l|l|l|l|l|l|l|l|l|l|}
\hline Original 5 euro & 45 & 402 & 871 & 1553 & 1938 & 7077 & 3406 & 793 & 6726 & 3769 & 49 \\
\hline Dirty 5 euro & 39 & 398 & 813 & 1456 & 1892 & 6981 & 3355 & 721 & 6451 & 3689 & 24 \\
\hline
\end{tabular}

\subsection{Support Vector Machine (SVM)}

The SVM is a classifier derived from statistical learning theory initially presented in [13]. It is based on the structural risk minimization principle (SRM). The main advantages of SVM when used for image classification problems are: (1) ability to work with

high dimensional data and (2) high generalization performance without the need to add a priori knowledge, even when the dimension of the input space is very high [14]. The problem that SVMs try to

solve is to find an optimal hyperplane that correctly

classifies data points by separating the points of two

classes as much as possible.

The SVM's linearly learned decision function $f(x)$ is described by weight vector $\mathrm{w}$, a threshold $\mathrm{b}$ and input patterns $\mathrm{x}$.

$$
f(x)=\operatorname{sign}(w \cdot x+b)
$$

Given a set of training vectors $\left\{S_{l}=\left(\left(x_{1}, y_{1}\right), . .,\left(x_{l}, y_{l}\right)\right), \quad x_{i} \in R^{l}\right\}$, equation (8) belonging to two separate classes, $\quad \mathrm{A}+\left(\mathrm{y}_{\mathrm{i}}=+1\right)$ and A$\left(y_{i}=-1\right)$, the SVM finds the hyperplane with maximum Euclidian distance from the training set. According to the SRM principle, there will be just one optimal hyperplane with the maximal margin $d$, defined as the sum of distances from the hyperplane to the closest points of the classes. This linear classifier threshold is the optimal separating hyperplane.

Graphically the support vectors are the set of points that mark the border of the class. This approach is valid whenever the set of points of the two classes are linearly separable. Nevertheless in real data this is usually not the case. In order to work with non-linear decision boundaries the key idea is to transform $\mathrm{x}_{\mathrm{i}}$ to a higher dimension space using a transformation function $\phi$, so that in this new space the samples can be linearly divided. SVM solve these problems using kernels. The relationship between the kernels function $K$ and $\phi$ is:

$k\left(x_{i}, x_{j}\right)=\phi\left(x_{i}\right) \phi\left(x_{j}\right)$

Intuitively, $k(x, y)$ represents the desired notion of similarity between data $\mathrm{x}$ and $\mathrm{y} . k(x, y)$ needs to satisfy a technical condition (Mercer condition) in order for $\phi$ to exist. An example of a kernel function is the Gaussian kernel, which is defined as

$$
k\left(x_{i}, x_{j}\right)=e^{-\left\|x_{i}-x_{j}\right\|^{2} / \sigma^{2}}
$$

When working with a Gaussian kernel, $\sigma$ represents the standard deviation, and ideally should represent the minimum distance between any two elements of two different classes. As it can be seen when constructing a SVM based on a Gaussian kernel, the only value that needs to be defined is $\sigma$. When working with kernels, in general it would not be possible to obtain w. Nevertheless SVM can be still be used. $\mathrm{N}_{\mathrm{S}}$ being the number of support vectors of the training set, the decision function can be expressed as

$f(x)=\sum_{i=1}^{N s_{s}} \alpha_{i} y_{i} \phi\left(x_{i}\right) \phi(x)+b=\sum_{i=1}^{N s_{s}} \alpha_{i} y_{i} k\left(x_{i}, x\right)+b$

two classes, SVM can be generalized to a set of C classes. In this case each one of the classes will be trained against the rest $\mathrm{C}-1$ classes, reducing the problem to a 2-class classification problem. 


\section{Proposed Paper Currency Recognition Method}

The proposed paper currency recognition method consists of the following steps:

I. The Wiener filter is initially applied to the original image to reduce the effect of noise. This technique has been used to increase the accuracy of the proposed method in recognizing dirty banknotes. The important point is that the severity of the increased darkness is almost the same on the whole surface of the paper currency.

II. The Fourier-Mellin transform is applied on the de-noised image obtained form step I. The main advantage of this technique is that the output image is invariant to translation, rotation and scale.

III. The original image is initially segmented into $\mathrm{n}$ segments, then the main diagonal of $\mathrm{N}$ matrix for each segment is calculated and used as features for paper currency recognition. It has been experimentally found that using segmentation, the performance of the proposed method would be increased.

IV. As it mentioned before, the SVM is a powerful method in the field of statistical learning theory which has been applied with success in pattern recognition applications. In this step, a SVM classifier has been used for paper currency recognition system. The calculated vectors are fed into the SVM classifier.

\section{Performance Evaluation}

Algorithms of the proposed method and existing techniques were implemented using MATLAB from Math Works, Inc. The performance of these techniques was evaluated using scaned real paper currency image.

To assess the performance of the proposed method, the technique was applied on a dataset of 150 banknotes of 101 different denominations, from 23 countries [15] (see Table 4). Among the database, $40 \%$ was some torn, worn and rotated banknotes. To find the best quantization level and the number of segments for the proposed approach, several experiments have been performed on various banknotes. The best number of quantization levels and number of segments have been chosen by considering the highest recognition rate. The results as shown in Table 5 indicate that quantizing the image of banknotes to 11 gray scales and dividing the paper currency image to 5 segments yields the best results.

The proposed technique was applied to both sides of the banknotes, and it could result $98.7 \%$ accuracy in recognizing paper currencies. Due to using the texture characteristic, the system recognizes the banknotes in any direction. To introduce a new denomination to the proposed system, the main diagonal values of the corresponding transition matrix of original paper currencies are required. It needs to be noted that only intact examples of paper currencies from each denomination are enough for training the system.
Table 4.

different banknotes from 23 countries

\begin{tabular}{|l|l|}
\hline Countries & Kinds of paper currency \\
\hline Afghanistan & $5,10,20,50,100,500$ \\
\hline Armenia & $500,1000,2000,5000,20000,50000$ \\
\hline Azerbaijan & $500,1000,10000,50000$ \\
\hline China & $10,20,50,100$ \\
\hline Euro & $5,10,20,50,100,200,500$ \\
\hline India & $5,20,100,500,1000$ \\
\hline Iran & $1000,2000,5000,10000,20000$ \\
\hline Iraq & $250,5000,10000,250000$ \\
\hline Japan & $1000,2000,5000,10000$ \\
\hline Jordan & $1,5,10,20$ \\
\hline Kazakhstan & $200,500,1000,5000$ \\
\hline Kuwait & $1 / 4,1,10,20$ \\
\hline Kyrgyzstan & $20,50,100,500,1000$ \\
\hline Pakistan & $5,50,100,500,1000$ \\
\hline Qatar & $1,5,10,50$ \\
\hline Russia & $10,50,100,500$ \\
\hline Saudi Arabia & $5,10,20,50$ \\
\hline Syria & $100,200,500,1000$ \\
\hline Turkey & $10,20,50,100$ \\
\hline Turkmenistan & $500,1000,5000,10000$ \\
\hline UAE & $5,10,20$ \\
\hline USA & $1,2,10,20$ \\
\hline Uzbekistan & $100,200,500$ \\
\hline
\end{tabular}

Table 5.

Recognition rates results form different quantization levels and segmentation of the paper currency images.

\begin{tabular}{|l|l|}
\hline Input Vector & Total Recognition Rate \\
\hline $1 \times 11$ & $37.4 \%$ \\
\hline $1 \times 52$ & $40.6 \%$ \\
\hline $2 \times 11$ & $44 \%$ \\
\hline $3 \times 11$ & $76.4 \%$ \\
\hline $4 \times 11$ & $93.9 \%$ \\
\hline $5 \times 11$ & $98.7 \%$ \\
\hline
\end{tabular}




\section{Conclusions}

In this paper a new paper currency recognition method using the Fourier-Mellin transform and SVM has been introduced.The main advantage of Fourier-Mellin transform technique is that the output image is invariant to translation, rotation and scale. The SVM is a powerful method in the field of statistical learning theory which has been applied with success in pattern recognition applications. Simulation results indicated proposed method achieved high accuracy in paper currency recognition. Performance results of applying the proposed methods on banknote denominations of 23 different countries indicate that the technique has $98.7 \%$ of accuracy.

\section{References}

[1] Debnath, K. K., Ahmed, S.U. and Shahjahan, M. 2010. A Paper Currency Recognition System Using Negatively Correlated Neural Network Ensemble. JOURNAL OF MULTIMEDIA, VOL. 5, NO. 6, 560-567.

[2] Hassanpour, H., Yaseri, A. and Ardeshir, G. 2002. Feature Extraction for Paper currency Recognition. In International symposium on signal processing and its applications (ISSPA), Sharjah, UAE, 1-4.

[3] Takeda, F. and Nishikage, T. 2000. Multiple kinds of paper currency recognition using neural network and application for Euro currency. In IEEE International Joint Conference on Neural Networks, Vol. 2, 143-147.

[4] Vila, A., Ferrer, N., Mantecon, J., Breton, D., and Garcia, J. F. 2006. Development of a fast and non-destructive procedure original and fake euro notes. Analytica Chimica Acta, 559, 257-263.

[5] Zhang, E. H., Jiang, B., Duan, J. H. and Bian, Z. Z. 2003. Research on paper currency recognition by neural networks, In Proceeding of the second international conference machine learning and cybernetics.

[6] Hassanpour, H., Farahabadi, P. M. 2009. Using Hidden Markov Models for paper currency recognition, Expert Systems With Applications , no 36, 10105-10111.

[7] Jae, Lim S. 1990. Two-dimensional signal and image processing. Englewood Cliffs, NJ: Prentice Hall.

[8] Gonzalez, R. C., and Woods, R. E. 2002. Digital image processing (2nd edition). Prentice Hall.

[9] Sena, A. D., Rocchesso, D. 2007. A fast Mellin and scale transform, EURASIP journal on Applied Signal Processing, Vol. 2007, 1-9.

[10] Cohen, L. 1993. The scale Representation, IEEE Trans on Signal Processing, Vol. 41, 3275-3291.

[11] Iosifescu, M. 1980. Finite Markov process and their applications. New York, NY: Wiley.

[12] Kim, M., Kim, D., and Lee, S. 2003. Face recognition using the embedded HMM with second-order blockspecific observations. Pattern Recognition, 36(11), 2723-2733.

[13] Cristianini, N. and Shawe-Taylor, J. 2000. An Introduction to Support Vector Machines. Cambridge University Press, Cambridge.

[14] Frias-Martinez, E., Sanchez, A. and Velez, J. 2006. Support vector machines versus multi layer perceptrons for efficient off-line signature recognition, Engineering Applications of Artificial Intelligence 19, 693-704.

[15]http://www.banknotes.com. 